TTP05-16

$\mathrm{SFB} / \mathrm{CPP}-05-44$

hep-ph/0508253

\title{
Electroweak corrections to hadronic photon production at large transverse momenta
}

\author{
Johann H. Kühn ${ }^{\dagger}$, A. Kulesza ${ }^{\ddagger}$, S. Pozzorini ${ }^{\S}$, M. Schulze \\ Institut für Theoretische Teilchenphysik, Universität Karlsruhe \\ D-76128 Karlsruhe, Germany
}

\begin{abstract}
:
We study the impact of electroweak radiative corrections on direct production of photons with high transverse momenta at hadron colliders. Analytic results for the weak one-loop corrections to the parton scattering reaction $\bar{q} q \rightarrow \gamma g$ and its crossed variants are presented. For the high-energy region, where the corrections are strongly enhanced by logarithms of $\hat{s} / M_{W}^{2}$, we derive simple asymptotic expressions which approximate the exact one-loop results with high precision. The dominant two-loop electroweak contributions are also calculated. Numerical results are presented for the LHC and the Tevatron. The corrections are negative and their size increases with transverse momentum. For the LHC, where transverse momenta of $2 \mathrm{TeV}$ or more can be reached, the size of the one- and two-loop effects amounts up to $-17 \%$ and $+3 \%$, respectively. At the Tevatron, with transverse momenta up to $400 \mathrm{GeV}$, the one-loop corrections do not exceed $-4 \%$ and the two-loop effects are negligible. Finally we compare the cross sections for hadronic production of photons and $Z$ bosons and find that the electroweak corrections have an important impact on their ratio.
\end{abstract}

August 2005

\footnotetext{
${ }^{\dagger}$ Johann.Kuehn@physik.uni-karlsruhe.de

$\ddagger$ ania@particle.uni-karlsruhe.de

§pozzorin@particle.uni-karlsruhe.de

^schulze@particle.uni-karlsruhe.de
} 


\section{Introduction}

The study of direct photon production, consisting of the QCD Compton process $g q \rightarrow \gamma q$ and the annihilation process $\bar{q} q \rightarrow \gamma g$, has always been an important topic in hadron colliders physics, both theoretically [1, 2] and experimentally [3. Since the photons do not fragment and can be clearly identified experimentally, direct photon production provides a much clearer probe of the hard-scattering dynamics than jet production processes. Therefore the study of large transverse momentum direct (prompt) photon production constitutes an important test of perturbative QCD and the point-like nature of quarks and gluons. Furthermore, the reaction contributes to background for many signals of new physics. Being embedded in the environment of hadronic collisions, the reaction necessarily involves hadronic physics, like parton distributions, and depends on the strong coupling constant. In turn, the cross section for direct production of photons, their tranverse momentum $\left(p_{\mathrm{T}}\right)$ and rapidity distributions can be used to gauge the parton distribution functions. Since the gluon distribution enters already at the leading order, the measurement of the direct photon production is an important means to constrain information on the gluon content of the proton [4. In particular, large transverse momentum production provides a unique opportunity for determination of gluon densities at large $x$.

Apart from the direct process, prompt photons can be also produced through a fragmentation process. However, most of the fragmentation contribution can be removed by applying an isolation criterion. The importance of the remaining contribution from fragmentation, after applying the isolation cut, is expected to decrease with higher $p_{\mathrm{T}}$. Moreover, background processes to isolated direct photon production, i.e. photon production through decays of neutral mesons $\left(\pi^{0}, \eta\right)$ coming from jet fragmentation, are shown to be less important at large $p_{\mathrm{T}}[5]$. To achieve reliable predictions at high $p_{\mathrm{T}}$, QCD corrections in next-to-leading order [2], are mandatory. The corrections can amount to several tens of percent [2, [5, 6], depending on the observable under consideration, value of $p_{\mathrm{T}}$, and details of the calculation such as jet definition or renormalization and factorization scales. The evaluation of next-to-next-to-leading order corrections involves two-loop virtual plus a variety of combined virtual plus real corrections and is a topic presently pursued by various groups [7.

For the experiments at the Large Hadron Collider (LHC) a new aspect comes into play. The high center-of-mass energy in combination with the enormous luminosity will allow to explore parton-parton scattering up to energies of several $\mathrm{TeV}$ and correspondingly production of gauge bosons with transverse momenta up to $2 \mathrm{TeV}$ or even beyond. In this region electroweak corrections from virtual weak boson exchange increase strongly, with the dominant terms in $L$-loop approximation being leading logarithms of the form $\alpha^{L} \log ^{2 L}\left(\hat{s} / M_{W}^{2}\right)$, next-to-leading logarithms of the form $\alpha^{L} \log ^{2 L-1}\left(\hat{s} / M_{W}^{2}\right)$, and so on. These corrections, also known as electroweak Sudakov logarithms, may well amount to several tens of percent. They have been studied in great detail for processes involving fermions in Refs. [8, 9]. Investigations 
on the dominant and the next-to-leading logarithmic terms are also available for reactions involving gauge and Higgs bosons [10, 11, 12. A recent survey of the literature on logarithmic electroweak corrections can be found in Ref. [13. The impact of these corrections at hadron colliders has been studied in Ref. 14. Specifically, hadronic $Z$-boson production at large $p_{\mathrm{T}}$ has been investigated in next-to-leading logarithmic approximation, including the two-loop terms [15, 16. Numerical results for the complete one-loop terms have been presented in Ref. [17 both for $Z$ and photon production.

It is the aim of this work to obtain an independent evaluation of the complete one-loop weak corrections to the photon production at large transverse momentum, and to present the full result in analytic form. At the partonic level the reactions $\bar{q} q \rightarrow \gamma g, g q \rightarrow \gamma q$ and $g \bar{q} \rightarrow \gamma \bar{q}$ with $q=u, d, c, s$ or $b$ have to be considered which are, however, trivially related by crossing and appropriate exchange of coupling constants. Much of the calculation proceeds in close similarity with the evaluation of $Z$ production presented in Ref. [16]. In fact, a significant part of the result can be obtained directly from the expressions published in Ref. [16]. We split the corrections into an "Abelian" and a "non-Abelian" component. We present analytic results for the exact one-loop corrections that permit to predict separately the various quark-helicity contributions. We also derive compact analytic expressions for the high-energy behaviour of the corrections. Here we include quadratic and linear logarithms as well as those terms that are not logarithmically enhanced at high energies but neglect all contributions of $\mathcal{O}\left(M_{W}^{2} / \hat{s}\right)$. The accuracy of this approximation is then discussed.

After convolution with parton distribution functions, radiatively corrected predictions for transverse momentum distributions of photons at hadron colliders are obtained. Concerning perturbative QCD, these predictions are based on the lowest order and thus proportional to $\alpha_{\mathrm{S}}$. To obtain realistic cross sections, higher-order QCD corrections would have to be included, in next-to-leading or even next-to-nextto-leading order.

The paper is organized as follows: In Sect. 2 the Born approximation, our conventions and the kinematics are introduced. Sect. 3] contains a detailed description of the radiative corrections. Sect. 3.1 is concerned with the renormalization procedure. In Sect. 3.2 our one-loop results are given in terms of expressions that had been introduced in Ref. [16] for the case of $Z$-boson production. Special attention is paid to results in the high-energy limit (Sect. 3.3), which can be cast into a compact form. Sect. 4 contains results for the dominant two-loop terms, i.e. the leading and next-to-leading Sudakov logarithms. In Sect. 5 a detailed discussion of numerical results is presented, both for $p p$ and $p \bar{p}$ collisions at $14 \mathrm{TeV}$ and $2 \mathrm{TeV}$, respectively. The leading and next-to-leading two-loop logarithmic terms are included in this numerical analysis. Sect. [6] contains a brief summary. 


\section{Preliminaries}

The transverse momentum $\left(p_{\mathrm{T}}\right)$ distribution of photons in the reaction $h_{1} h_{2} \rightarrow \gamma+$ jet is given by

$$
\frac{\mathrm{d} \sigma^{h_{1} h_{2}}}{\mathrm{~d} p_{\mathrm{T}}}=\sum_{i, j} \int_{0}^{1} \mathrm{~d} x_{1} \int_{0}^{1} \mathrm{~d} x_{2} \theta\left(x_{1} x_{2}-\hat{\tau}_{\min }\right) f_{h_{1}, i}\left(x_{1}, \mu^{2}\right) f_{h_{2}, j}\left(x_{2}, \mu^{2}\right) \frac{\mathrm{d} \hat{\sigma}^{i j}}{\mathrm{~d} p_{\mathrm{T}}},
$$

where $\hat{\tau}_{\text {min }}=4 p_{\mathrm{T}}^{2} / s$ and $\sqrt{s}$ is the collider energy. The indices $i, j$ denote initial state partons $(q, \bar{q}, g)$ and $f_{h_{1}, i}\left(x, \mu^{2}\right), f_{h_{2}, j}\left(x, \mu^{2}\right)$ are the corresponding parton distribution functions. The partonic cross section for the subprocess $i j \rightarrow \gamma k$ is denoted by $\hat{\sigma}^{i j}$ and the sum in (11) runs over all $i, j$ combinations corresponding to the subprocesses

$$
\bar{q} q \rightarrow \gamma g, \quad q \bar{q} \rightarrow \gamma g, \quad g q \rightarrow \gamma q, \quad q g \rightarrow \gamma q, \quad \bar{q} g \rightarrow \gamma \bar{q}, \quad g \bar{q} \rightarrow \gamma \bar{q}
$$

with $q=u, d, c, s$ or $b$. The Mandelstam variables for the subprocess $i j \rightarrow \gamma k$ are defined in the standard way

$$
\hat{s}=\left(p_{i}+p_{j}\right)^{2}, \quad \hat{t}=\left(p_{i}-p_{\gamma}\right)^{2}, \quad \hat{u}=\left(p_{j}-p_{\gamma}\right)^{2} .
$$

The momenta $p_{i}, p_{j}, p_{k}$ and $p_{\gamma}$ of the partons are massless. In terms of $x_{1}, x_{2}, p_{\mathrm{T}}$ and the collider energy $\sqrt{s}$ we have

$$
\hat{s}=x_{1} x_{2} s, \quad \hat{t}=-\frac{\hat{s}}{2}(1-\cos \theta), \quad \hat{u}=-\frac{\hat{s}}{2}(1+\cos \theta),
$$

with $\cos \theta=\sqrt{1-4 p_{\mathrm{T}}^{2} / \hat{s}}$ corresponding to the cosine of the angle between the momenta $p_{i}$ and $p_{\gamma}$ in the partonic center-of-mass frame.

The angular and the $p_{\mathrm{T}}$ distribution for the unpolarized partonic subprocess $i j \rightarrow \gamma k$ read

$$
\frac{\mathrm{d} \hat{\sigma}^{i j}}{\mathrm{~d} \cos \theta}=\frac{1}{32 \pi N_{i j} \hat{s}} \bar{\sum}\left|\mathcal{M}^{i j}\right|^{2}
$$

and

$$
\frac{\mathrm{d} \hat{\sigma}^{i j}}{\mathrm{~d} p_{\mathrm{T}}}=\frac{p_{\mathrm{T}}}{8 \pi N_{i j} \hat{s}|\hat{t}-\hat{u}|}\left[\bar{\sum}\left|\mathcal{M}^{i j}\right|^{2}+(\hat{t} \leftrightarrow \hat{u})\right],
$$

where $\bar{\sum}=\frac{1}{4} \sum_{\text {pol }} \sum_{\text {col }}$ involves the sum over polarization and color as well as the average factor $1 / 4$ for initial-state polarization. The factor $1 / N_{i j}$ in (15)-(6) , with $N_{\bar{q} q}=N_{q \bar{q}}=N_{\mathrm{c}}^{2}, N_{g q}=N_{q g}=N_{\bar{q} g}=N_{g \bar{q}}=N_{\mathrm{c}}\left(N_{\mathrm{c}}^{2}-1\right)$, and $N_{\mathrm{c}}=3$, accounts for the initial-state colour average.

In the following we present analytic results for the unpolarized squared matrix elements $\bar{\sum}\left|\mathcal{M}^{\bar{q} q}\right|^{2}$ for the $\bar{q} q \rightarrow \gamma g$ process. Corresponding results for the other 


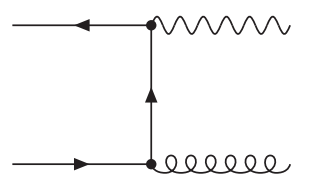

$(\mathrm{t} 1)$

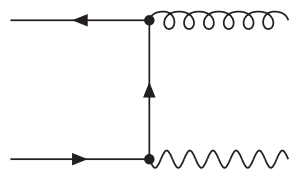

$(\mathrm{t} 2)$

Figure 1: Tree-level Feynman diagrams for the process $\bar{q} q \rightarrow \gamma g$.

processes in (2) are easily obtained by means of CP symmetry and crossing transformations 16 .

The lowest-order contribution results from the tree diagrams depicted in Fig. 1] and reads

$$
\bar{\sum}\left|\mathcal{M}_{0}^{\bar{q} q}\right|^{2}=16 \pi^{2} \alpha \alpha_{\mathrm{S}}\left(N_{\mathrm{c}}^{2}-1\right) Q_{q}^{2} \frac{\hat{t}^{2}+\hat{u}^{2}}{\hat{t} \hat{u}}
$$

where $Q_{u}=2 / 3, Q_{d}=-1 / 3, \alpha=e^{2} /(4 \pi)$ and $\alpha_{\mathrm{S}}=g_{\mathrm{S}}^{2} /(4 \pi)$ are the quark electric charges, and the electromagnetic and the strong coupling constants, respectively.

\section{One-loop corrections}

In this section, we present analytic results for the one-loop weak corrections to the process $\bar{q} q \rightarrow \gamma g$. They are closely related to those presented in Ref. [16] for the process $\bar{q} q \rightarrow Z g$. In fact, the one-loop diagrams for $\bar{q} q \rightarrow \gamma g$, see Fig. 2] are analogous to the diagrams for $\bar{q} q \rightarrow Z g$. Hence a large subset of corrections to $\bar{q} q \rightarrow \gamma g$ can be obtained directly from the results for $\bar{q} q \rightarrow Z g$ by setting $M_{Z}$ to zero. However, the renormalization counterterms need to be calculated separately for the $\bar{q} q \rightarrow \gamma g$ process.

Similarly as in the $Z$-boson production case [16, we do not include electromagnetic corrections in the full one-loop result. The renormalized weak corrections are infrared finite. To obtain the results of Ref. [16], we assumed all quarks to be massless, neglected diagrams involving couplings of quarks to Higgs bosons or wouldbe-Goldstone bosons and omitted quark-mixing effects. The calculation of Ref. [16] was performed at the level of matrix elements and allows for a full control over polarization effects. The one-loop amplitude was split into two parts according to the structure of the gauge group generators in front of each term: Abelian (characteristic for Abelian theories) and non-Abelian (originating from the non-commutativity of weak interactions). Tensor loop integrals, appearing in the expressions for oneloop corrections were reduced to scalar integrals by means of the Passarino-Veltman technique. Assuming zero quark masses led to mass singularities of collinear nature, present at the level of individual integrals. However, these cancel between various scalar integrals, making it possible to write the final result in terms of finite combi- 


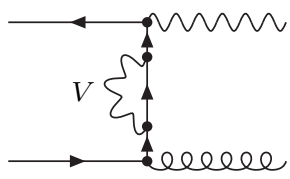

(s1)

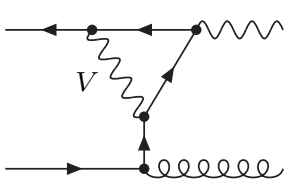

(v3)

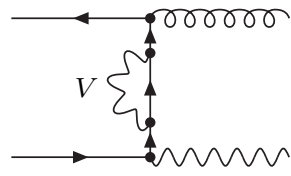

$(\mathrm{s} 2)$

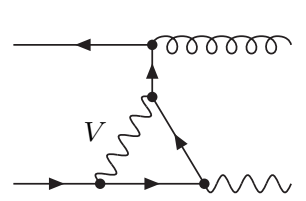

( v4)

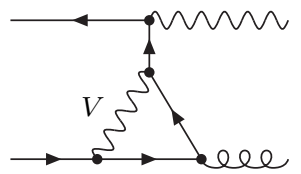

(v1)

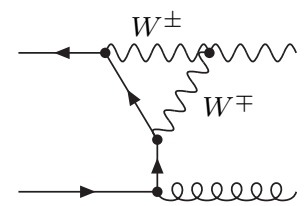

(v5)

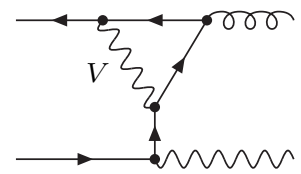

$(\mathrm{v} 2)$

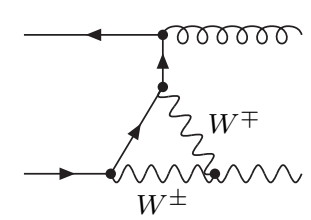

(v6)

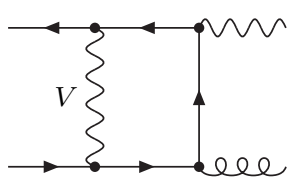

(b1)

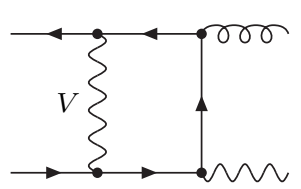

(b2)

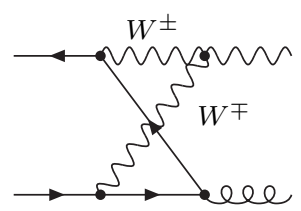

(b3)

Figure 2: One-loop Feynman diagrams for the process $\bar{q} q \rightarrow \gamma g$. The diagrams v5, v6 and b3 involve only charged weak bosons, $W^{ \pm}$, whereas the other diagrams receive contributions from neutral and charged weak bosons, $V=Z, W^{ \pm}$.

nations of the integrals. Further details concerning the calculation can be found in Ref. [16].

In the following, only these contributions are presented in detail that cannot be directly derived from Ref. [16] by setting $M_{Z}$ to zero. We start discussing renormalization in the on-shell scheme, in Sect. 3.1. The complete one-loop corrections and their asymptotic behaviour are then presented in Sect. 3.2 and Sect. 3.3. respectively.

\subsection{Renormalization}

The renormalization of the $\bar{q} q \rightarrow \gamma g$ process is provided by the $\gamma q \bar{q}$ counterterm. ${ }^{1}$ This can be written, similarly to the $Z q \bar{q}$ counterterm [16], as

$$
\not m=-\mathrm{i} e \gamma^{\mu} \sum_{\lambda=\mathrm{R}, \mathrm{L}} \omega_{\lambda}\left[Q_{q}\left(\delta Z_{q_{\lambda}}+\delta C_{q_{\lambda}}^{\mathrm{A}}\right)+T_{q_{\lambda}}^{3} \delta C_{q_{\lambda}}^{\mathrm{N}}\right]
$$

\footnotetext{
${ }^{1}$ Same as for the case of the $\bar{q} q \rightarrow Z g$ process [16], the counterterm contributions associated with the $g q \bar{q}$ vertex and the quark propagator cancel.
} 
with $\omega_{\mathrm{R}}=\left(1+\gamma^{5}\right) / 2$ and $\omega_{\mathrm{L}}=\left(1-\gamma^{5}\right) / 2$. As in Ref. [16],

$$
\delta Z_{q_{\lambda}}=\frac{\alpha}{4 \pi} \sum_{V=\mathrm{Z}, \mathrm{W}^{ \pm}}\left(I^{V} I^{\bar{V}}\right)_{q_{\lambda}}\left[\frac{3}{2}-\frac{A_{0}\left(M_{V}^{2}\right)}{M_{V}^{2}}\right]
$$

are the wave-function renormalization constants of massless chiral quarks. The coupling factors in (9) read

$$
\left(I^{Z} I^{Z}\right)_{q_{\lambda}}=\left(\frac{c_{\mathrm{W}}}{s_{\mathrm{W}}} T_{q_{\lambda}}^{3}-\frac{s_{\mathrm{W}}}{c_{\mathrm{W}}} \frac{Y_{q_{\lambda}}}{2}\right)^{2}, \quad \sum_{V=W^{ \pm}}\left(I^{V} I^{\bar{V}}\right)_{q_{\lambda}}=\frac{\delta_{\lambda \mathrm{L}}}{2 s_{\mathrm{W}}^{2}}
$$

with the shorthands $c_{\mathrm{W}}=\cos \theta_{\mathrm{W}}$ and $s_{\mathrm{W}}=\sin \theta_{\mathrm{W}}$ for the weak mixing angle $\theta_{\mathrm{W}}$. $Y_{q_{\lambda}} / 2=Q_{q}-T_{q_{\lambda}}^{3}$ and $T_{q_{\lambda}}^{3}$ is the weak isospin of chiral quarks, i.e. $T_{u_{\lambda}}^{3}=\delta_{\lambda \mathrm{L}} / 2$ and $T_{d_{\lambda}}^{3}=-\delta_{\lambda \mathrm{L}} / 2$. The remaining counterterm contributions in (8) are split in an Abelian part, $\delta C_{q_{\lambda}}^{\mathrm{A}}$, proportional to the tree-level coupling $Q_{q}$ and a non-Abelian part, $\delta C_{q_{\lambda}}^{\mathrm{N}}$, proportional to the weak isospin $T_{q_{\lambda}}^{3}$. In the following we adopt the on-shell renormalization scheme [18], with $c_{\mathrm{w}}^{2}=1-s_{\mathrm{W}}^{2}=M_{W}^{2} / M_{Z}^{2}$ and the electromagnetic coupling constant defined in the Thompson limit, i.e. at the scale zero. In this scheme, the Abelian counterterm $\delta C_{q_{\lambda}}^{\mathrm{A}}$ vanishes owing to Ward identities [18],

$$
\delta C_{q_{\lambda}}^{\mathrm{A}}=\frac{1}{2}\left(\delta Z_{A A}+\frac{s_{\mathrm{W}}}{c_{\mathrm{W}}} \delta Z_{Z A}+\frac{\delta e^{2}}{e^{2}}\right)=0 .
$$

The expressions for the counterterms $\delta Z_{A A}, \delta Z_{Z A}$ and $\delta e^{2} / e^{2}$ can be found in Ref. [18. As well known, the on-shell counterterm $\delta e^{2} / e^{2}$ contains large logarithms of light-fermion masses, which are responsible for the running of $\alpha$ from the scale zero to the characteristic scale of the process. However, owing to the Ward identity, for the case of on-shell photon production these logarithms are cancelled by corresponding terms present in $\delta Z_{A A}$, see (11). This justifies our choice of $\alpha$ at the scale zero as input parameter.

The non-Abelian counterterm reads

$$
\delta C_{q_{\lambda}}^{\mathrm{N}}=-\frac{1}{2 s_{\mathrm{W}} c_{\mathrm{W}}} \delta Z_{Z A},
$$

where

$$
\delta Z_{Z A}=2 \frac{\sum_{\mathrm{T}}^{\mathrm{AZ}}(0)}{M_{Z}^{2}}=\frac{\alpha}{4 \pi} \frac{4 c_{\mathrm{W}}}{s_{\mathrm{W}}}\left[\bar{\Delta}_{\mathrm{UV}}-\log \left(\frac{M_{W}^{2}}{M_{Z}^{2}}\right)\right]
$$

with

$$
\bar{\Delta}_{\mathrm{UV}}=1 / \varepsilon-\gamma_{\mathrm{E}}+\log (4 \pi)+\log \left(\frac{\mu_{\mathrm{R}}^{2}}{M_{Z}^{2}}\right)
$$

in $D=4-2 \varepsilon$ dimensions. Here $\mu_{\mathrm{R}}$ denotes the scale of dimensional regularization. 


\section{$3.2 \quad$ Result}

The complete $\mathcal{O}\left(\alpha^{2} \alpha_{\mathrm{S}}\right)$ result for the $\bar{q} q \rightarrow \gamma g$ process can be written as

$$
\begin{aligned}
& \bar{\sum}\left|\mathcal{M}_{1}^{\bar{q} q}\right|^{2}=8 \pi^{2} \alpha \alpha_{\mathrm{S}}\left(N_{\mathrm{c}}^{2}-1\right) \\
& \times \sum_{\lambda=\mathrm{R}, \mathrm{L}}\left\{Q_{q}^{2}\left[H_{0}+\frac{\alpha}{2 \pi} \sum_{V=\mathrm{Z}, \mathrm{W}^{ \pm}}\left(I^{V} I^{\bar{V}}\right)_{q_{\lambda}} H_{1}^{\mathrm{A}}\left(M_{V}^{2}\right)\right]\right. \\
& \left.+T_{q_{\lambda}}^{3} Q_{q}\left[2 H_{0} \delta C_{q_{\lambda}}^{\mathrm{N}}+\frac{\alpha}{2 \pi} \frac{1}{s_{\mathrm{W}}^{2}} H_{1}^{\mathrm{N}}\left(M_{W}^{2}\right)\right]\right\}
\end{aligned}
$$

where $H_{0}=\left(\hat{t}^{2}+\hat{u}^{2}\right) /(\hat{t} \hat{u})$ is the Born contribution and $\delta C_{q_{\lambda}}^{\mathrm{N}}$ is the counterterm specified in Sect. 3.1. The remaining functions $H_{1}^{\mathrm{A} / \mathrm{N}}\left(M_{V}^{2}\right)$ represent the Abelian (A) and non-Abelian (N) contributions resulting from the loop diagrams (see Fig. 2) involving virtual weak bosons, $V=Z, W^{ \pm}$, and the fermionic wave-function renormalization constants (9). The above result is analogous to the one presented in Ref. [16] for the $\bar{q} q \rightarrow Z g$ process. In particular, the functions $H_{1}^{\mathrm{A} / \mathrm{N}}\left(M_{V}^{2}\right)$ for the $\bar{q} q \rightarrow \gamma g$ process can be obtained from the corresponding functions for the $\bar{q} q \rightarrow Z g$ process [16] by setting the mass of the external $Z$ boson equal to zero. ${ }^{2}$ This simple relation between the loop corrections to the processes $\bar{q} q \rightarrow \gamma g$ and $\bar{q} q \rightarrow Z g$ is due to the fact that, for $Z$ production, the longitudinal contributions to the $Z$-boson polarization sum vanish. This is a consequence of the Ward identities presented in Sect. 3.6 of Ref. [16].

\subsection{High-energy limit}

Let us now consider the high-energy region, $\hat{s} / M_{W}^{2} \gg 1$, where the weak corrections are strongly enhanced by logarithms of the form $\log \left(\hat{s} / M_{W}^{2}\right)$. As in Ref. [16] we derive compact analytic expressions that describe the asymptotic high-energy behaviour of the one-loop corrections, $H_{1}^{\mathrm{A} / \mathrm{N}}\left(M_{V}^{2}\right)$, to next-to-next-to-leading logarithmic (NNLL) accuracy. This approximation includes double and single logarithms as well as those contributions that are not logarithmically enhanced at high energies. The NNLL expressions for $H_{1}^{\mathrm{N}}\left(M_{W}^{2}\right), H_{1}^{\mathrm{A}}\left(M_{W}^{2}\right)$ and $H_{1}^{\mathrm{A}}\left(M_{Z}^{2}\right)$, obtained by means of general results for the high-energy limit of one-loop integrals [19], have the form

$$
H_{1}^{\mathrm{A} / \mathrm{N}}\left(M_{V}^{2}\right) \stackrel{\mathrm{NNLL}}{=} \operatorname{Re}\left[g_{0}^{\mathrm{A} / \mathrm{N}}\left(M_{V}^{2}\right) \frac{\hat{t}^{2}+\hat{u}^{2}}{\hat{t} \hat{u}}+g_{1}^{\mathrm{A} / \mathrm{N}}\left(M_{V}^{2}\right) \frac{\hat{t}^{2}-\hat{u}^{2}}{\hat{t} \hat{u}}+g_{2}^{\mathrm{A} / \mathrm{N}}\left(M_{V}^{2}\right)\right] .
$$

In principle, the functions $g_{i}^{\mathrm{A} / \mathrm{N}}\left(M_{V}^{2}\right)$ for the $\bar{q} q \rightarrow \gamma g$ process can be obtained from the corresponding functions for the $\bar{q} q \rightarrow Z g$ process [16] by setting the mass of the

\footnotetext{
${ }^{2}$ The functions $H_{1}^{\mathrm{A} / \mathrm{N}}\left(M_{V}^{2}\right)$ presented in Ref. [16] consist of linear combinations of loop integrals $J_{0}, \ldots, J_{14}$. We observe that the integrals $J_{2}, J_{8}$ and $J_{10}$ are logarithmically singular for $M_{Z} \rightarrow 0$. However the corresponding coefficients are proportional to $M_{Z}^{2}$. Thus the contributions of such loop integrals vanish for $M_{Z} \rightarrow 0$.
} 
external $Z$ boson equal to zero. However, this must be done with care since the $Z$-mass dependence of the $g_{i}^{\mathrm{A} / \mathrm{N}}\left(M_{V}^{2}\right)$ functions in Ref. [16] is not shown explicitly. For the case of photon production we find that the functions $g_{i}^{\mathrm{A}}\left(M_{V}^{2}\right)$ with $i=0,1,2$ and $g_{j}^{\mathrm{N}}\left(M_{W}^{2}\right)$ with $j=1,2$ are the same as in Ref. [16]. Instead, for the non-Abelian function $g_{0}^{\mathrm{N}}\left(M_{W}^{2}\right)$ we obtain

$$
\begin{aligned}
g_{0}^{\mathrm{N}}\left(M_{W}^{2}\right)= & 2\left[\bar{\Delta}_{\mathrm{UV}}-\log \left(\frac{M_{W}^{2}}{M_{Z}^{2}}\right)\right]+\log ^{2}\left(\frac{-\hat{s}}{M_{W}^{2}}\right)-\log ^{2}\left(\frac{-\hat{t}}{M_{W}^{2}}\right)-\log ^{2}\left(\frac{-\hat{u}}{M_{W}^{2}}\right) \\
& +\log ^{2}\left(\frac{\hat{t}}{\hat{u}}\right)-\frac{3}{2}\left[\log ^{2}\left(\frac{\hat{t}}{\hat{s}}\right)+\log ^{2}\left(\frac{\hat{u}}{\hat{s}}\right)\right]-2 \pi^{2}
\end{aligned}
$$

Here the logarithmic terms are the same as in the $Z$-production case whereas the constants are different. In particular, the coefficient of the $\pi^{2}$ term is different and no term of the form $\pi / \sqrt{3}$ is present in (17). Logarithms with negative arguments in (17) are defined through the usual i $\varepsilon$ prescription, $\hat{r} \rightarrow \hat{r}+\mathrm{i} \varepsilon$ for $\hat{r}=\hat{s}, \hat{t}, \hat{u}$. Assuming, as in Ref. [16], that one of the Mandelstam invariants is positive and the other two are negative, for the real part of (17) we obtain

$$
\begin{aligned}
\operatorname{Re} g_{0}^{\mathrm{N}}\left(M_{W}^{2}\right)= & 2\left[\bar{\Delta}_{\mathrm{UV}}-\log \left(\frac{M_{W}^{2}}{M_{Z}^{2}}\right)\right]+\log ^{2}\left(\frac{|\hat{s}|}{M_{W}^{2}}\right)-\log ^{2}\left(\frac{|\hat{t}|}{M_{W}^{2}}\right)-\log ^{2}\left(\frac{|\hat{u}|}{M_{W}^{2}}\right) \\
& +\log ^{2}\left(\frac{|\hat{t}|}{|\hat{u}|}\right)-\frac{3}{2}\left[\log ^{2}\left(\frac{|\hat{t}|}{|\hat{s}|}\right)+\log ^{2}\left(\frac{|\hat{u}|}{|\hat{s}|}\right)\right]-\frac{\pi^{2}}{2} \theta(-\hat{s}) .
\end{aligned}
$$

This result applies directly to the $\bar{q} q \rightarrow \gamma g$ process, where $\hat{s}>0, \hat{t}<0$ and $\hat{u}<0$. In this case $\theta(-\hat{s})=0$. The $\theta$ term becomes non-vanishing when (18) is translated, by means of permutations of the Mandelstam invariants (crossing transformations), to the processes in (2) that involve gluons in the initial state.

\section{Two-loop corrections}

In the $\mathrm{TeV}$ energy region, the logarithmic electroweak corrections are very large. The one-loop contributions can amount to tens of percent and also the two-loop logarithmic terms can reach the level of several percent, see e.g. Ref. 16]. Such higher-order contributions are thus mandatory for precise theoretical predictions. In the following, we present the two-loop corrections to the process $\bar{q} q \rightarrow \gamma g$ to next-to-leading logarithmic (NLL) accuracy. For a discussion of the calculation we refer to Ref. [15], where the same class of corrections has been computed for the process $\bar{q} q \rightarrow Z g$.

The unpolarized squared matrix element for $\bar{q} q \rightarrow \gamma g$, including NLL terms up to the two-loop level, has the general form

$$
\bar{\sum}\left|\mathcal{M}_{2}^{\bar{q} q}\right|^{2}=8 \pi^{2} \alpha \alpha_{\mathrm{S}}\left(N_{\mathrm{c}}^{2}-1\right) \frac{\hat{t}^{2}+\hat{u}^{2}}{\hat{t} \hat{u}}\left[A^{(0)}+\left(\frac{\alpha}{2 \pi}\right) A^{(1)}+\left(\frac{\alpha}{2 \pi}\right)^{2} A^{(2)}\right] .
$$


The Born contribution reads

$$
A^{(0)}=2 Q_{q}^{2}
$$

At one loop, the NLL part consists of double- and single-logarithmic terms and reads

$$
A^{(1)} \stackrel{\mathrm{NLL}}{=}-\sum_{\lambda=\mathrm{L}, \mathrm{R}} Q_{q}\left[Q_{q}\left(C_{q_{\lambda}}^{\mathrm{ew}}-Q_{q}^{2}\right)\left(\mathrm{L}_{\hat{s}}^{2}-3 \mathrm{~L}_{\hat{s}}\right)+\frac{1}{s_{\mathrm{W}}^{2}} T_{q_{\lambda}}^{3}\left(\mathrm{~L}_{\hat{t}}^{2}+\mathrm{L}_{\hat{u}}^{2}-\mathrm{L}_{\hat{s}}^{2}\right)\right] .
$$

Here we used the shorthand $\mathrm{L}_{\hat{r}}^{k}=\log ^{k}\left(|\hat{r}| / M_{W}^{2}\right)$ for the logarithms and $C_{q_{\lambda}}^{\text {ew }}=$ $Y_{q_{\lambda}}^{2} /\left(4 c_{\mathrm{w}}^{2}\right)+C_{q_{\lambda}} / s_{\mathrm{w}}^{2}$ are the eigenvalues of the electroweak Casimir operator for quarks, with $C_{q_{\mathrm{L}}}=3 / 4$ and $C_{q_{\mathrm{R}}}=0$. Only weak corrections are included in (21). This result is obtained in the $M_{Z}=M_{W}$ approximation and is consistent with the leading- and next-to-leading logarithmic part of the one-loop asymptotic expressions $^{3}$ presented in Sect. 3. At two loops, for the complete electroweak NLL corrections $^{4}$ we obtain

$$
\begin{aligned}
A^{(2) \stackrel{\mathrm{NLL}}{=}} \sum_{\lambda=\mathrm{L}, \mathrm{R}}\left\{\frac { 1 } { 2 } ( Q _ { q } C _ { q _ { \lambda } } ^ { \mathrm { ew } } + \frac { 1 } { s _ { \mathrm { W } } ^ { 2 } } T _ { q _ { \lambda } } ^ { 3 } ) \left[Q_{q} C_{q_{\lambda}}^{\mathrm{ew}}\left(\mathrm{L}_{\hat{s}}^{4}-6 \mathrm{~L}_{\hat{s}}^{3}\right)\right.\right. \\
\left.+\frac{1}{s_{\mathrm{W}}^{2}} T_{q_{\lambda}}^{3}\left(\mathrm{~L}_{\hat{t}}^{4}+\mathrm{L}_{\hat{u}}^{4}-\mathrm{L}_{\hat{s}}^{4}\right)\right]+\frac{T_{q_{\lambda}}^{3} Y_{q_{\lambda}}}{8 s_{\mathrm{W}}^{4}}\left(\mathrm{~L}_{\hat{t}}^{4}+\mathrm{L}_{\hat{u}}^{4}-\mathrm{L}_{\hat{s}}^{4}\right) \\
\left.+\frac{1}{6} Q_{q}\left[Q_{q}\left(\frac{b_{1}}{c_{\mathrm{W}}^{2}}\left(\frac{Y_{q_{\lambda}}}{2}\right)^{2}+\frac{b_{2}}{s_{\mathrm{W}}^{2}} C_{q_{\lambda}}\right)+\frac{1}{s_{\mathrm{W}}^{2}} T_{q_{\lambda}}^{3} b_{2}\right] \mathrm{L}_{\hat{s}}^{3}\right\},
\end{aligned}
$$

where $b_{1}=-41 /\left(6 c_{\mathrm{w}}^{2}\right)$ and $b_{2}=19 /\left(6 s_{\mathrm{w}}^{2}\right)$ are the one-loop $\beta$-function coefficients associated with the $\mathrm{U}(1)$ and $\mathrm{SU}(2)$ couplings, respectively. Eq. (23) has been derived from the general results of Refs. [12, 20, for leading- and next-to-leading electroweak two-loop logarithms.

\section{$5 \quad$ Numerical results}

In this section, we present numerical predictions for photon production at high transverse momenta, both at the partonic and hadronic level. We also compare the cross sections for hadronic photon and $Z$-boson production and study the impact of the corrections on their ratio. The lowest order (LO) and the next-to-leading-order (NLO) predictions for photon production result from (7) and (15), respectively.

\footnotetext{
${ }^{3}$ The correspondence between (21) and the one-loop expressions of Sect. 3 is easily seen by means of the relation$$
C_{q_{\lambda}}^{\mathrm{ew}}-Q_{q}^{2}=\sum_{V=\mathrm{Z}, \mathrm{W}^{ \pm}}\left(I^{V} I^{\bar{V}}\right)_{q_{\lambda}}
$$

${ }^{4}$ At the two-loop level the purely weak corrections cannot be isolated from the complete electroweak corrections in a gauge-invariant way. One must thus consider the combination of weak and electromagnetic virtual corrections. The latter are regularized by means of a fictitious photon mass $\lambda=M_{\mathrm{W}}$. This approach is discussed in detail in Ref. [15.
} 
The next-to-leading-logarithmic ${ }^{5}$ (NLL) approximation, at one loop, corresponds to the contributions (20) and (21). The next-to-next-to-leading-logarithmic (NNLL) predictions, also at one loop, are based on the asymptotic expressions (16)-(18) combined with the exact result (13) for the counterterm. Our best predictions, to next-to-next-to-leading-order (NNLO), include the exact NLO contributions combined with the leading and next-to-leading two-loop terms (23).

The hadronic cross sections are obtained using LO MRST2001 parton distribution functions (PDFs) [21]. We choose $p_{T}^{2}$ as the factorization scale and, similarly as the scale at which the running strong coupling constant is evaluated. We also adopt the value $\alpha_{\mathrm{S}}\left(M_{Z}^{2}\right)=0.13$ and use the one-loop running expression for $\alpha_{\mathrm{S}}\left(\mu^{2}\right)$, in accordance with the LO PDF extraction method of the MRST collaboration. We use the following values of parameters [22]: $\alpha=1 / 137.0, M_{Z}=91.19 \mathrm{GeV}$, $M_{W}=80.39 \mathrm{GeV}$ and $s_{\mathrm{w}}^{2}=1-c_{\mathrm{w}}^{2}=1-M_{W}^{2} / M_{Z}^{2}$.

We begin by investigating NLO, NLL and NNLL relative corrections to the partonic (unpolarized) differential cross section $\mathrm{d} \hat{\sigma}^{i j} / \mathrm{d} \cos \theta[$ see (15)]. To this end we define

$$
\hat{\mathcal{R}}_{\mathrm{NLO} / \mathrm{LO}}^{i j}=\frac{\mathrm{d} \hat{\sigma}_{\mathrm{NLO}}^{i j} / \mathrm{d} \cos \theta}{\mathrm{d} \hat{\sigma}_{\mathrm{LO}}^{i j} / \mathrm{d} \cos \theta}-1
$$

and similarly $\hat{\mathcal{R}}_{\mathrm{NLL} / \mathrm{NLO}}^{i j}$ and $\hat{\mathcal{R}}_{\mathrm{NNLL} / \mathrm{NLO}}^{i j}$. These ratios, calculated at $\cos \theta=0$, are displayed as a function of $\sqrt{\hat{s}}$ in Fig. 3. We consider four processes: $\bar{u} u \rightarrow \gamma g$ (Fig. 3a), $\bar{d} d \rightarrow \gamma g$ (Fig. 3b), $g u \rightarrow \gamma u$ (Fig. 3r), $g d \rightarrow \gamma d$ (Fig. 31 d). The NLO corrections are negative, their absolute size growing with the partonic energy $\sqrt{\hat{s}}$. At $\sqrt{\hat{s}}=4 \mathrm{TeV}$ the corrections reach $-15 \%$ for the $\bar{u} u$ and $g u$ channels, $-23 \%$ for the $\bar{d} d$, and $-28 \%$ for the $g d$ channel. The NLO correction is well approximated by the NLL result: for $\sqrt{\hat{s}} \geq 200 \mathrm{GeV}$ the approximation is accurate up to $2 \%$ in all channels. Even better an approximation of the one-loop weak correction is provided by the NNLL result. In $\bar{u} u$ and $\bar{d} d$ channels the NNLL approximation differs from the NLO result by less than $1 \%$ in the entire region under consideration, whereas for the $g u$ and $g d$ channels the quality of the approximation is at the level of (or better than) one permille. The absolute LO and NLO cross section and the $\hat{\mathcal{R}}^{i j}$ ratios for specific values of $\cos \theta, \sqrt{\hat{s}}$ and different collision channels are listed in Table 1 .

The transverse momentum distribution $\mathrm{d} \sigma / \mathrm{d} p_{\mathrm{T}}$ at the LHC is shown in Fig. 4. We display separately the absolute values of the LO, NLO, NLL and NNLL differential cross sections (Fig. 4a) and the relative correction wrt. the LO result for the NLO, NLL and NNLL distributions (Fig. 4 $\mathrm{b}$ ). The relative correction wrt. the LO is now defined as

$$
\mathcal{R}_{\mathrm{NLO} / \mathrm{LO}}^{\mathrm{had}}=\frac{\mathrm{d} \sigma_{\mathrm{NLO}} / \mathrm{d} p_{\mathrm{T}}}{\mathrm{d} \sigma_{\mathrm{LO}} / \mathrm{d} p_{\mathrm{T}}}-1
$$

for the NLO case, and similarly for the NLL and the NNLL cross sections. The quality of the NLL and NNLL high-energy approximations of the NLO result is shown in more detail in Fig. 4k. The contribution provided by the NLO correction is negative

\footnotetext{
${ }^{5}$ At the NLL level, angular-dependent logarithms are treated as in Ref. [15.
} 


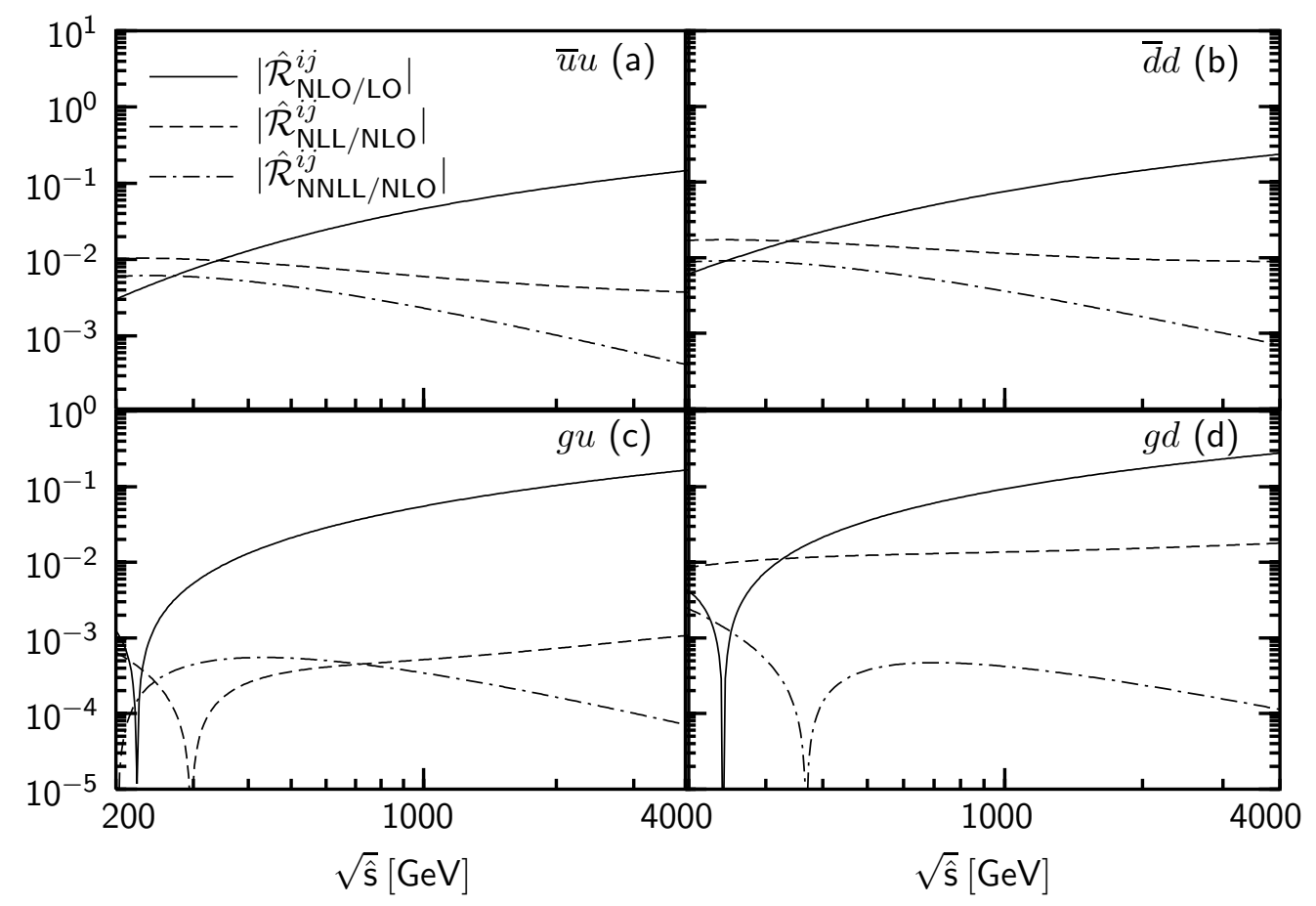

Figure 3: Relative one-loop corrections to the partonic differential cross sections $\mathrm{d} \hat{\sigma}^{i j} / \mathrm{d} \cos \theta$ at $\cos \theta=0$ for (a) $\bar{u} u$ channel, (b) $\bar{d} d$ channel, (c) $g u$ channel, (d) $g d$ channel. The solid, dashed and dot-dashed lines denote the modulus of the $\hat{\mathcal{R}}$ ratios, as defined in the text, for the full NLO cross section, the NLL approximation and the NNLL approximation of the one-loop cross section, respectively.

and increases in size with $p_{\mathrm{T}}$. It ranges from $-6 \%$ at $p_{\mathrm{T}}=500 \mathrm{GeV}$ up to $-17 \%$ at

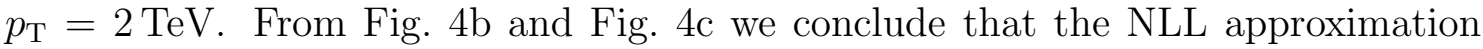
works well, differing from the full NLO prediction by about 3 permille at low $p_{\mathrm{T}}$ and by less than 1 permille at $p_{\mathrm{T}} \sim 2 \mathrm{TeV}$. The quality of the NNLL approximation is very good, at the level of accuracy of $10^{-3}$ or better in the entire $p_{\mathrm{T}}$ range. In Fig. 5 we show the relative size of the corrections in the NLO approximation $\left(\mathcal{R}_{\mathrm{NLO} / \mathrm{LO}}^{\mathrm{had}}\right.$, solid line), and in the approximation which includes the next-to-leading logarithmic two-loop terms $\left(\mathcal{R}_{\mathrm{NNLO} / \mathrm{LO}}^{\mathrm{had}}\right.$, dotted line). These additional two-loop terms are positive, their size increasing with $p_{\mathrm{T}}$. At $p_{\mathrm{T}}=2 \mathrm{TeV}$ they amount to $3 \%$, yielding the total (i.e. together with the NLO) correction to the LO of $-14 \%$.

To underline the relevance of these effects, in Fig. [ we present the relative NLO and NNLO corrections for the cross section, integrated over $p_{\mathrm{T}}$ starting from $p_{\mathrm{T}}=p_{\mathrm{T}}^{\text {cut }}$, as a function of $p_{\mathrm{T}}^{\text {cut }}$. This is compared with the statistical error, defined as $\Delta \sigma_{\text {stat }} / \sigma=1 / \sqrt{N}$ with $N=\mathcal{L} \times \sigma_{\mathrm{LO}}$. We assume a total integrated luminosity $\mathcal{L}=300 \mathrm{fb}^{-1}$ for the LHC [23]. It is clear from Fig. [6, that the size of the one-loop (two-loop) corrections is much bigger than (comparable to) the statistical error. 


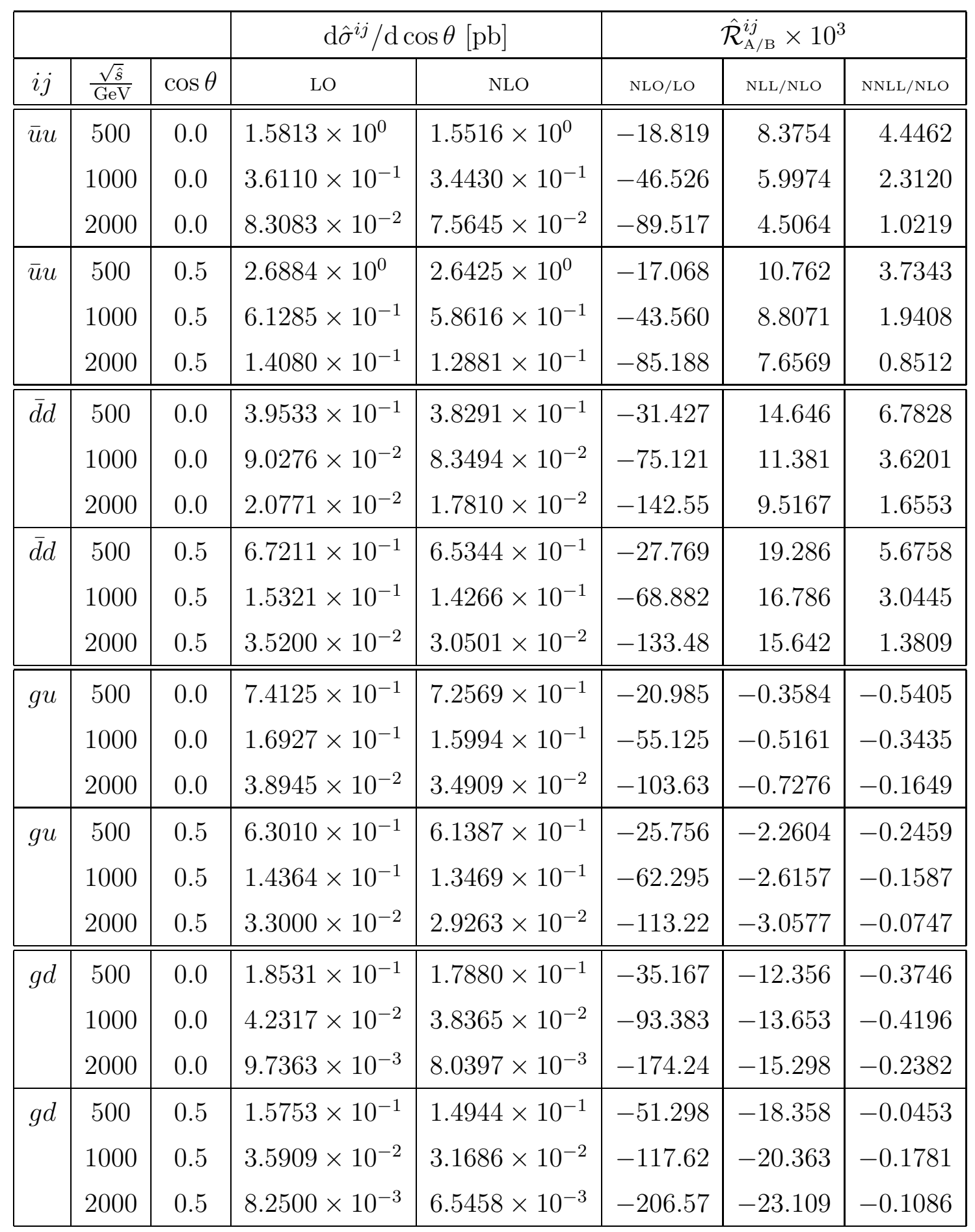

Table 1: Absolute value of the LO and NLO partonic differential cross section $\mathrm{d} \hat{\sigma}^{i j} / \mathrm{d} \cos \theta$ and the ratios $\hat{\mathcal{R}}_{\mathrm{NLO} / \mathrm{LO}}^{i j}, \hat{\mathcal{R}}_{\mathrm{NLL} / \mathrm{NLO}}^{i j}$ and $\hat{\mathcal{R}}_{\mathrm{NNLL} / \mathrm{NLO}}^{i j}$ in permille, for the partonic processes $\bar{u} u \rightarrow \gamma g, \bar{d} d \rightarrow \gamma g, g u \rightarrow \gamma u$ and $g d \rightarrow \gamma d$. The running strong coupling $\alpha_{\mathrm{S}}\left(\mu^{2}\right)$ is taken at the scale $\mu^{2}=p_{\mathrm{T}}^{2}=\left(1-\cos ^{2} \theta\right) \hat{s} / 4$. 


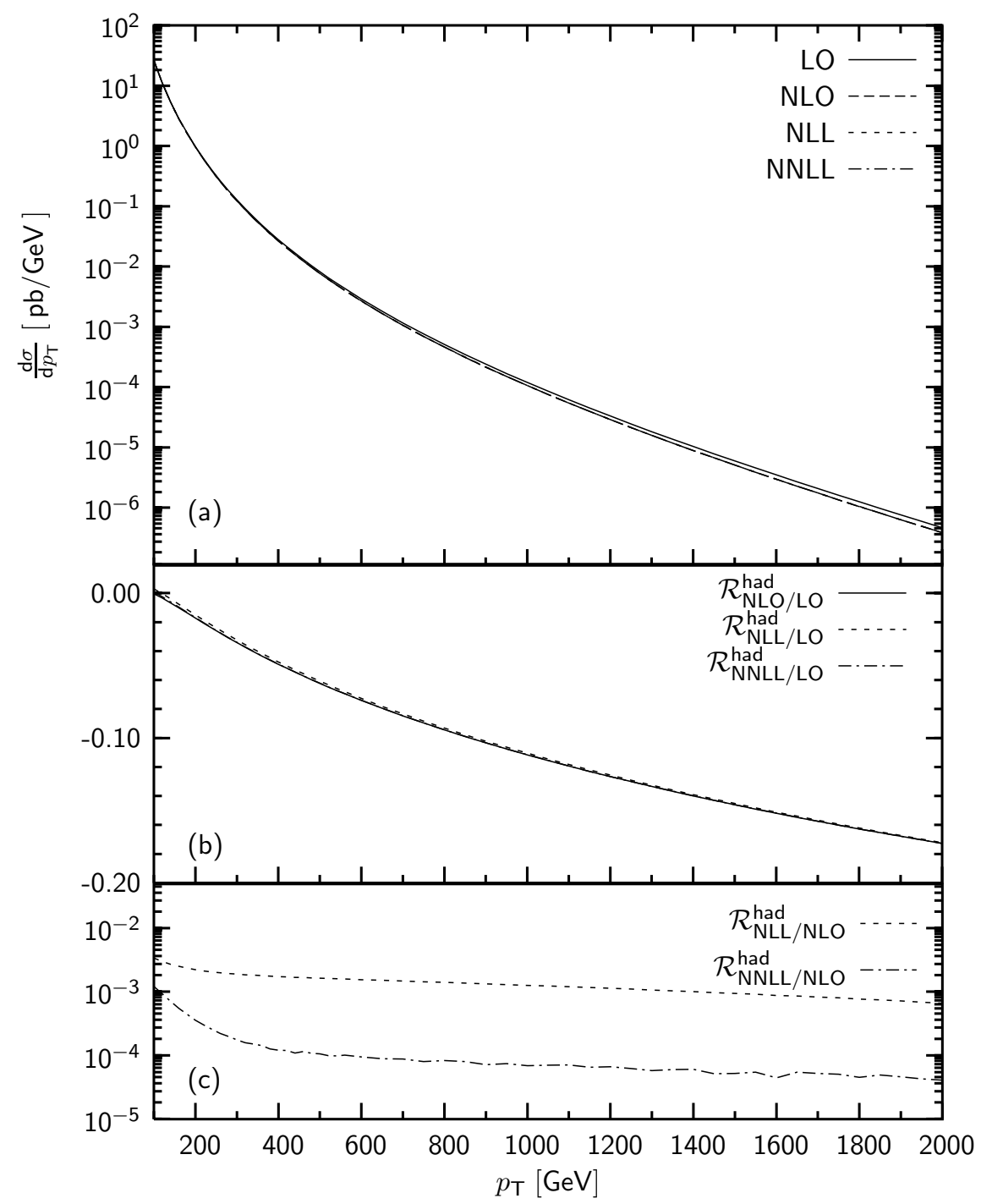

Figure 4: Transverse momentum distribution for $p p \rightarrow \gamma j$ at $\sqrt{s}=14 \mathrm{TeV}$. (a) LO (solid), NLO (dashed), NLL (dotted) and NNLL (dot-dashed) predictions. (b) Relative NLO (solid), NLL (dotted) and NNLL (dot-dashed) weak correction wrt. the LO distribution. (c) NLL (dotted) and NNLL (dot-dashed) approximations compared to the full NLO result. 


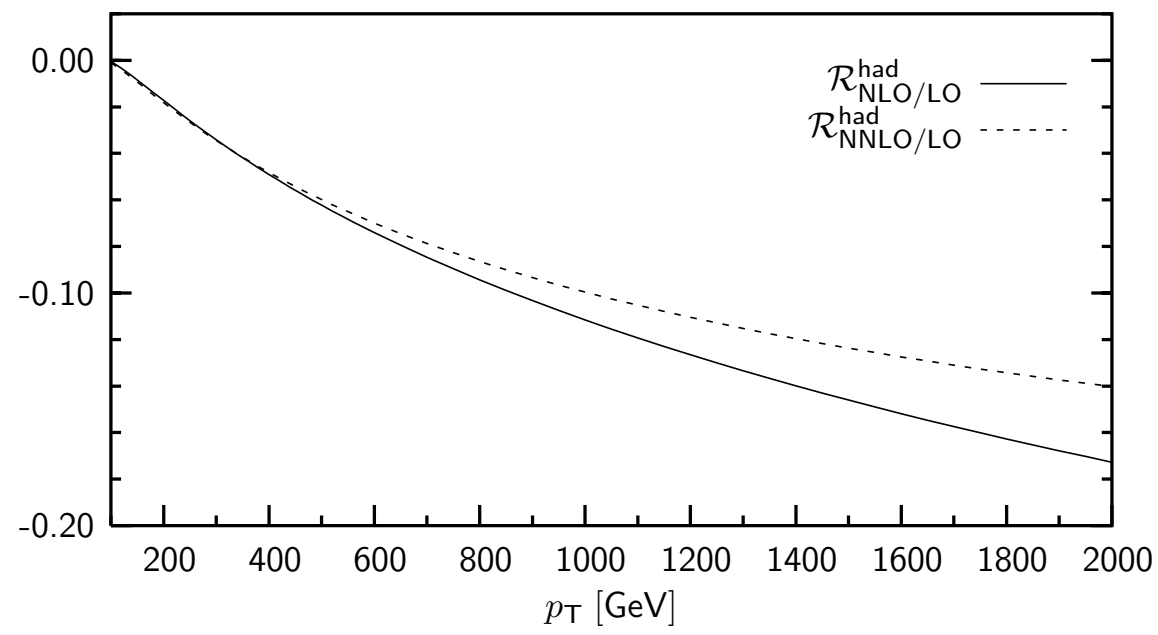

Figure 5: Relative NLO (solid) and NNLO (dotted) corrections to the $p_{\mathrm{T}}$ distribution for $p p \rightarrow \gamma j$ at $\sqrt{s}=14 \mathrm{TeV}$.

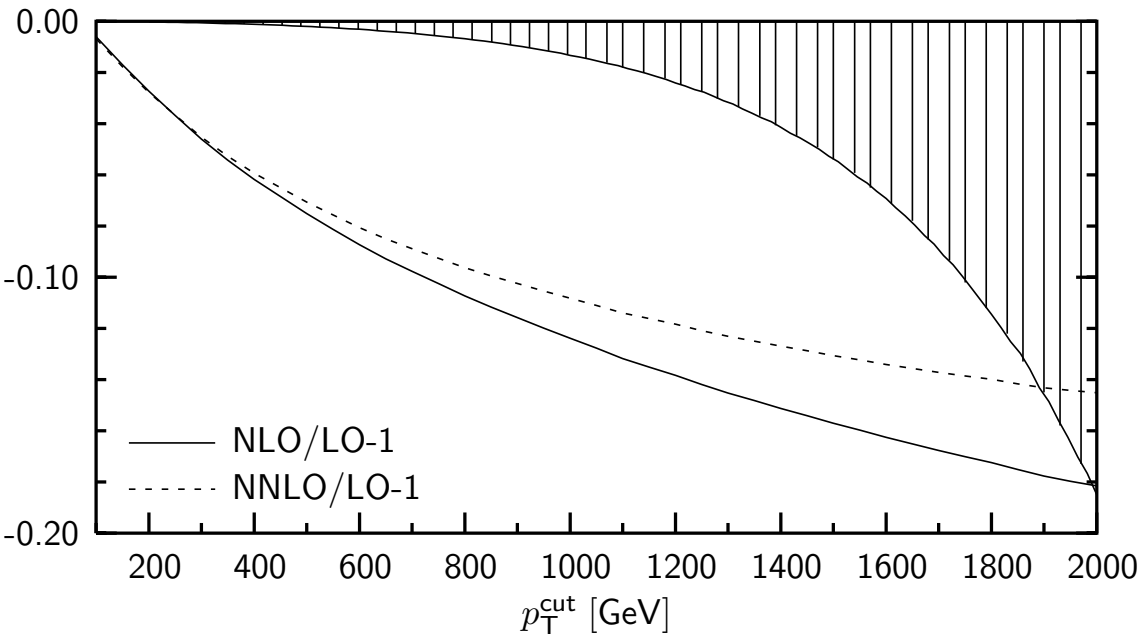

Figure 6: Relative NLO (solid) and NNLO (dotted) corrections wrt. the LO prediction and statistical error (shaded area) for the unpolarized integrated cross section for $p p \rightarrow \gamma j$ at $\sqrt{s}=14 \mathrm{TeV}$ as a function of $p_{\mathrm{T}}^{\text {cut }}$. 


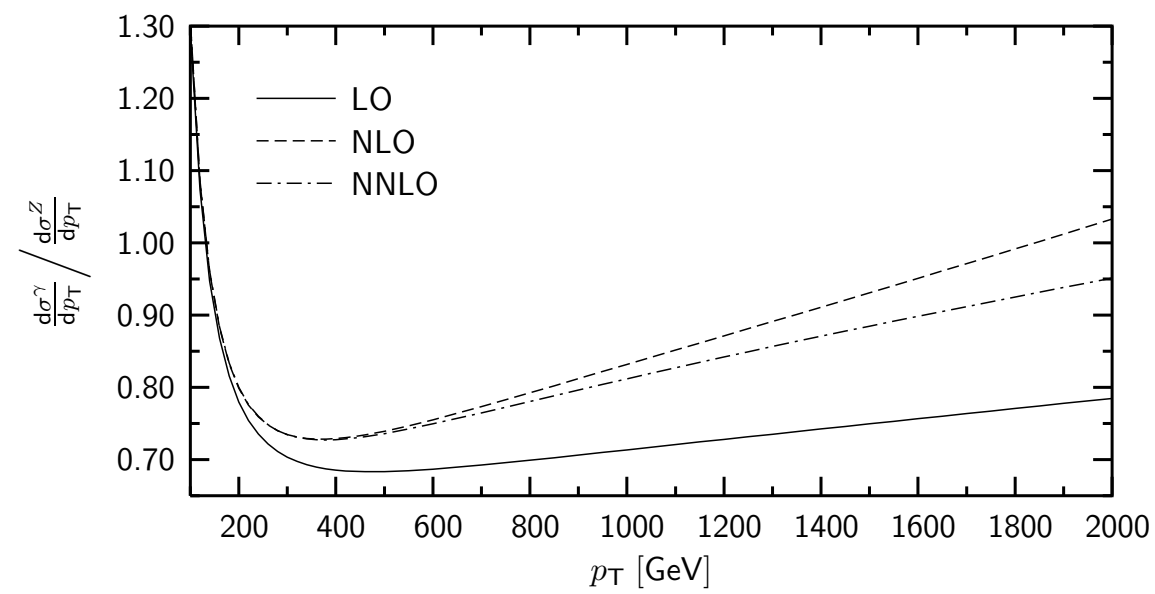

Figure 7: Ratio of the transverse momentum distributions for the processes $p p \rightarrow \gamma j$ and $p p \rightarrow Z j$ at $\sqrt{s}=14 \mathrm{TeV}: \mathrm{LO}$ (solid), NLO(dashed) and NNLO (dot-dashed) predictions.

In Fig. 17, we plot the ratio of the $p_{\mathrm{T}}$ distributions of photons and $Z$ bosons. The latter is computed within the $\overline{\mathrm{MS}}$ scheme using the same input parameters as in Ref. [16]. Such ratio is expected to be less sensitive to theoretical errors than the distributions themselves, since many uncertainties such as the scale at which $\alpha_{\mathrm{S}}$ is calculated or the choice of PDFs cancel to a large extend in the ratio. Moreover, due to a similar cancellation mechanism, the ratio should remain stable against QCD corrections. From Fig. [7]we observe that the weak corrections modify the production ratio considerably. The effect is the strongest at high $p_{\mathrm{T}}$. In this region, the LO photon cross section is smaller than the cross section for $Z$ boson production by about $25 \%$. The relatively large NLO corrections for $Z$ production, as compared to $\gamma$ production, cause the full NLO production rates to become equal at the highest $p_{\mathrm{T}}$ considered here, i.e. $p_{\mathrm{T}} \sim 2 \mathrm{TeV}$. The two-loop corrections modify the ratio and lead to a few percent decrease at high $p_{\mathrm{T}}$.

We perform a similar analysis for direct photon production at high transverse momentum at the Tevatron. In Fig. 8 we show the transverse momentum distribution (Fig. $8 \mathrm{a}$ ), the relative size of the corrections (Fig. 8 b) and the quality of the one-loop NLL and NNLL approximations (Fig. 8k). The effects of weak corrections are generally much smaller for the case of the Tevatron than the LHC, with the NLO corrections not exceeding $-4 \%$ at the highest $p_{\mathrm{T}}$ considered, i.e. at $p_{\mathrm{T}} \sim 400 \mathrm{GeV}$. In Fig. 9 the relative size of the corrections to the integrated cross section is compared with the statistical error expected for an integrated luminosity $\mathcal{L}=11 \mathrm{fb}^{-1}[24$. At the energies of the Fermilab collider, the NLO weak correction is of the order of the statistical error and we conclude it should be taken into account when considering precision measurements. The two-loop terms turn out to be negligible. The ratio of the $p_{\mathrm{T}}$ distributions of photons and $Z$ bosons is shown in Fig. 10, Since the weak 
corrections to the $Z$ and $\gamma$ production at the Tevatron are moderate, their effect on the ratio is fairly small and stays within a few percent range for all values of $p_{\mathrm{T}}$ considered here.

Our numerical results, both for the case of the LHC and the Tevatron, are in agreement with the results presented in figures of Ref. [17].

\section{Summary}

In this work we present one-loop weak corrections to the direct production of photons with large transverse momenta at hadron colliders. Analytical results are given for the parton subprocess $\bar{q} q \rightarrow \gamma g$ and its crossed versions. We also present approximate expressions valid in the high-energy region, $\hat{s} \gg M_{W}^{2}$, where the weak corrections are enhanced by logarithms of $\hat{s} / M_{W}^{2}$. The complete high-energy approximation discussed in this paper includes all large logarithms as well as those terms that are not logarithmically enhanced. This approximation is in very good agreement with the complete one-loop result. We also calculate the two-loop electroweak corrections in the next-to-leading logarithmic approximation.

The corrections are then evaluated numerically for proton-antiproton collisions at $2 \mathrm{TeV}$ (Tevatron) and proton-proton collisions at $14 \mathrm{TeV}$ (LHC) in the region of large tranverse momentum $\left(p_{\mathrm{T}}\right)$. The corrections are negative and their size increases with $p_{\mathrm{T}}$. At the Tevatron, transverse momenta up to $400 \mathrm{GeV}$ will be explored and the weak corrections may reach up to $-4 \%$. At the LHC, transverse momenta of $2 \mathrm{TeV}$ or more are within the reach. In this region the one-loop corrections are significant, about $-17 \%$, and even the dominant two-loop logarithmic terms must be included in precise predictions. Finally we compare the cross sections for photon and $Z$-boson production and find that the electroweak corrections have a considerable impact on their ratio at the $\mathrm{LHC}$.

\section{Acknowledgements}

This work was supported in part by BMBF Grant No. 05HT4VKA/3 and by the Deutsche Forschungsgemeinschaft (Sonderforschungsbereich Transregio SFB/TR-9 "Computational Particle Physics"). M. S. would like to acknowledge financial support from the Graduiertenkolleg "Hochenergiephysik und Teilchenastrophysik". 


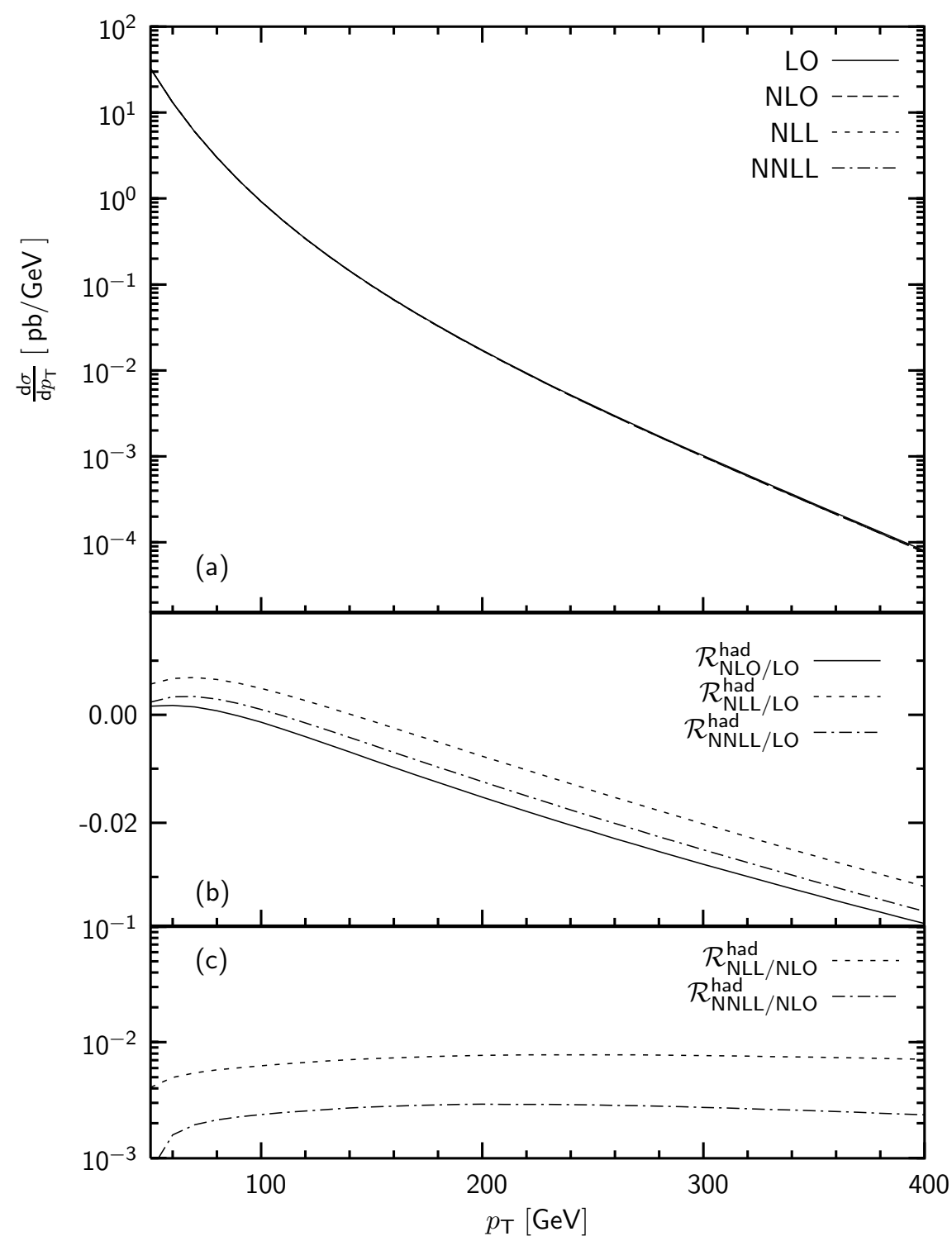

Figure 8: Transverse momentum distribution for $p \bar{p} \rightarrow \gamma j$ at $\sqrt{s}=2 \mathrm{TeV}$. (a) LO (solid), NLO (dashed), NLL (dotted) and NNLL (dot-dashed) predictions. (b) Relative NLO (solid), NLL (dotted) and NNLL (dot-dashed) weak correction wrt. the LO distribution. (c) NLL (dotted) and NNLL (dot-dashed) approximations compared to the full NLO result. 


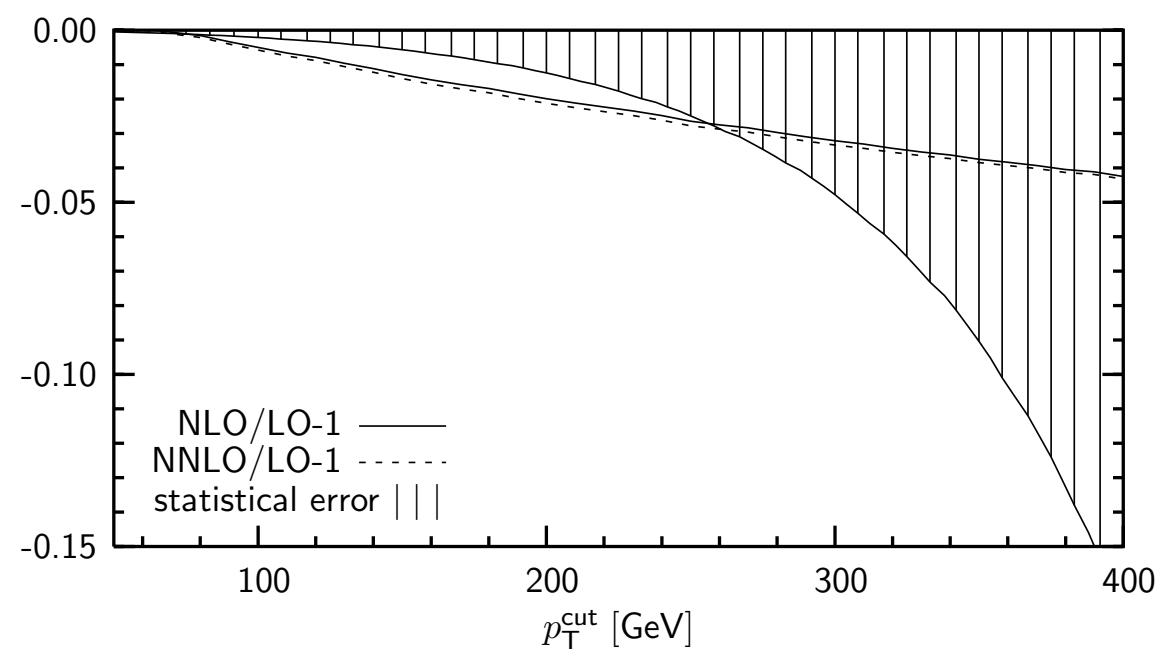

Figure 9: Relative NLO (solid) and NNLO (dotted) corrections wrt. the LO and statistical error (shaded area) for the unpolarized integrated cross section for $p \bar{p} \rightarrow \gamma j$ at $\sqrt{s}=2 \mathrm{TeV}$ as a function of $p_{\mathrm{T}}^{\text {cut }}$.

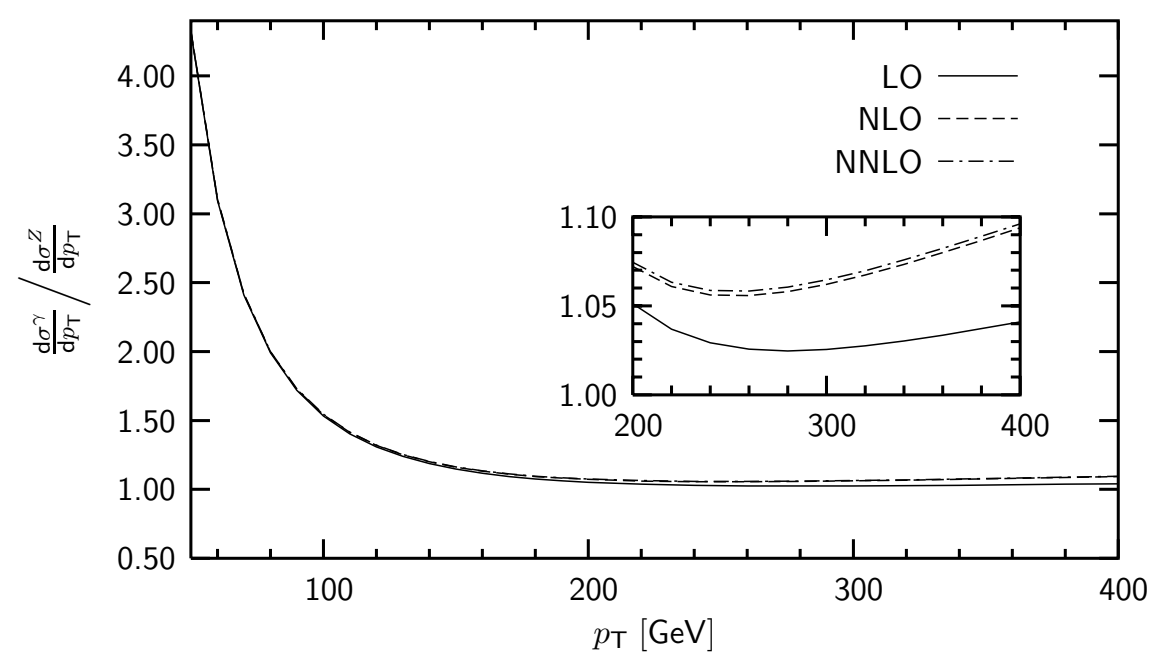

Figure 10: Ratio of the transverse momentum distributions for the processes $p \bar{p} \rightarrow \gamma j$ and $p \bar{p} \rightarrow Z j$ at $\sqrt{s}=2 \mathrm{TeV}$ : LO (solid), NLO(dashed) and NNLO (dot-dashed) predictions. 


\section{References}

[1] H. Fritzsch and P. Minkowski, Phys. Lett. B 69 (1977) 316.

Also, for a review see: J. F. Owens, Rev. Mod. Phys. 59 (1987) 465.

[2] P. Aurenche et al., Phys. Lett. B 140 (1984) 87;

P. Aurenche et al., Nucl. Phys. B 297 (1988) 661;

H. Baer, J. Ohnemus and J. F. Owens, Phys. Lett. B 234 (1990) 127; Phys.

Rev. D 42 (1990) 61;

E. L. Berger and J. w. Qiu, Phys. Lett. B 248 (1990) 371; Phys. Rev. D 44 (1991) 2002;

P. Aurenche et al., Nucl. Phys. B 399 (1993) 34;

L. E. Gordon and W. Vogelsang, Phys. Rev. D 48 (1993) 3136; Phys. Rev. D 50 (1994) 1901;

S. Catani et al., JHEP 0205 (2002) 028 hep-ph/0204023.

[3] F. Abe et al. [CDF Collaboration], Phys. Rev. Lett. 73 (1994) 2662 [Erratumibid. 74 (1995) 1891];

D. Acosta et al. [CDF Collaboration]; Phys. Rev. D 65 (2002) 112003 hep-ex/0201004;

B. Abbott et al. [D0 Collaboration], Phys. Rev. Lett. 84 (2000) 2786 hep-ex/9912017;

V. M. Abazov et al. [D0 Collaboration], Phys. Rev. Lett. 87 (2001) 251805 hep-ex/0106026;

L. Apanasevich et al. [Fermilab E706 Collaboration], Phys. Rev. Lett. 81 (1998) 2642 hep-ex/9711017; Phys. Rev. D 70 (2004) 092009 hep-ex/0407011;

G. Ballocchi et al. [UA6 Collaboration], Phys. Lett. B $4 \mathbf{3 6}$ (1998) 222.

For a review of older data, see: W. Vogelsang and M. R. Whalley, J. Phys. G 23 (1997) A1.

[4] P. Aurenche, R. Baier and M. Fontannaz, Phys. Rev. D 42 (1990) 1440;

W. Vogelsang and A. Vogt, Nucl. Phys. B 453 (1995) 334 hep-ph/9505404.

[5] A. Kumar et al., Phys. Rev. D 67 (2003) 014016.

[6] J. Huston et al., Phys. Rev. D 51 (1995) 6139 hep-ph/9501230|;

P. Aurenche et al., Eur. Phys. J. C 9 (1999) 107 hep-ph/9811382.

[7] A. Gehrmann-De Ridder, T. Gehrmann and E. W. N. Glover, Nucl. Phys. Proc. Suppl. 135 (2004) 97 hep-ph/0407023;

S. Weinzierl, hep-ph/0408278.

[8] J. H. Kühn and A. A. Penin, hep-ph/9906545;

J. H. Kühn, A. A. Penin and V. A. Smirnov, Eur. Phys. J. C 17 (2000) 97 [hep-ph/9912503]; 
J. H. Kühn et al., Nucl. Phys. B 616 (2001) 286 [hep-ph/0106298];

B. Feucht, J. H. Kühn and S. Moch, Phys. Lett. B 561 (2003) 111 hep-ph/0303016;

B. Feucht et al., Phys. Rev. Lett. 93 (2004) 101802 hep-ph/0404082;

B. Jantzen et al., hep-ph/0504111.

[9] S. Pozzorini, Nucl. Phys. B 692 (2004) 135 hep-ph/0401087.

[10] A. Denner and S. Pozzorini, Eur. Phys. J. C 18 (2001) 461 [hep-ph/0010201]; Eur. Phys. J. C 21 (2001) 63 [hep-ph/0104127].

[11] S. Pozzorini, doctoral thesis, Universität Zürich, 2001, hep-ph/0201077.

[12] A. Denner, M. Melles and S. Pozzorini, Nucl. Phys. B 662 (2003) 299 hep-ph/0301241.

[13] W. Hollik et al., Acta Phys. Polon. B 35 (2004) 2533 hep-ph/0501246|.

[14] E. Accomando, A. Denner and S. Pozzorini, Phys. Rev. D 65 (2002) 073003 [hep-ph/0110114];

W. Hollik and C. Meier, Phys. Lett. B 590 (2004) 69 hep-ph/0402281;

E. Accomando, A. Denner and A. Kaiser, Nucl. Phys. B 706 (2005) 325 hep-ph/0409247;

S. Moretti, M. R. Nolten and D. A. Ross, hep-ph/0503152.

[15] J. H. Kühn, A. Kulesza, S. Pozzorini and M. Schulze, Phys. Lett. B 609 (2005) 277 hep-ph/0408308.

[16] J. H. Kühn, A. Kulesza, S. Pozzorini and M. Schulze, hep-ph/0507178.

[17] E. Maina, S. Moretti and D. A. Ross, Phys. Lett. B 593 (2004) 143 [Erratumibid. B 614 (2005) 216] hep-ph/0403050).

[18] A. Denner, Fortsch. Phys. 41 (1993) 307.

[19] M. Roth and A. Denner, Nucl. Phys. B 479 (1996) 495 hep-ph/9605420.

[20] M. Melles, Phys. Rev. D 63 (2001) 034003 [hep-ph/0004056];

[21] A. D. Martin, R. G. Roberts, W. J. Stirling and R. S. Thorne, Phys. Lett. B 531 (2002) 216 hep-ph/0201127.

[22] S. Eidelman et al. [Particle Data Group], Phys. Lett. B 592 (2004) 1.

[23] V. A. Mitsou, hep-ph/0004161

[24] Department of Energy Review Committee Report on the Run II Luminosity Performance of the Fermilab Tevatron, October 28-31 2002, http://www-bd.fnal.gov/doereview02/Run_Il_lum_review_Final.pdf 